\title{
IgG4-related Skin Lesions in a Patient with IgG4-related Chronic Sclerosing Dacryoadenitis and Sialoadenitis
}

\author{
Yasushi Kakuchi ${ }^{1}$, Kazunori Yamada ${ }^{1}$, Yasunori Suzuki ${ }^{1}$, Naoko Ito ${ }^{2}$, Kunimasa Yagi ${ }^{2}$, \\ Masami Matsumura ${ }^{3}$, Masakazu Yamagishi ${ }^{2}$, Hisanori Umehara ${ }^{4}$, Yoh Zen ${ }^{5}$, \\ Minoru Hasegawa ${ }^{6}$, Kazuhiko Takehara ${ }^{6}$ and Mitsuhiro Kawano ${ }^{1}$
}

\begin{abstract}
We describe a 60-year-old man with IgG4-related chronic sclerosing dacryoadenitis and sialoadenitis associated with lymphoplasmacytic and eosinophilic infiltration in erythematous nodules. Physical examination revealed left eye extrusion and small itchy nodules on the scalp and neck. The serum IgG level was 1,570 $\mathrm{mg} / \mathrm{dL}, \mathrm{IgG} 4463 \mathrm{mg} / \mathrm{dL}$ (29.5\%), and IgE 4,554 IU/mL. Lacrimal gland biopsy disclosed prominent infiltrates of IgG4-positive plasma cells and scattered eosinophilic infiltrates with fibrosis, consistent with IgG4related disease. A skin biopsy of a cutaneous nodule demonstrated that the infiltrated plasma cells around arterioles or venules in the deep dermis and subcutaneous fat tissue were strongly positive for IgG4. Although the swollen lacrimal and parotid gland and itchy subcutaneous erythematous nodules improved rapidly with oral prednisolone at a dose of $20 \mathrm{mg}$ per day, the skin, lacrimal, and parotid lesions deteriorated simultaneously during steroid tapering and improved after increasing the dosage. As skin lesions are easy to biopsy, further study of the skin manifestations of IgG4-related disease will be important in further clarifying the clinical spectrum, pathophysiology and response to therapy of this disorder.
\end{abstract}

Key words: IgG4-related disease, cutaneous lymphoid infiltrate, IgG4-related chronic sclerosing dacryoadenitis and sialoadenitis

(Intern Med 50: 1465-1469, 2011)

(DOI: 10.2169/internalmedicine.50.5239)

\section{Introduction}

After the establishment of the entity of autoimmune pancreatitis (AIP) (1,2), a variety of associated extra-pancreatic lesions have been reported including those of the lacrimal glands, salivary glands, lungs, kidneys, liver, bile duct, retroperitoneum, breast, aorta, pituitary gland, and prostate (3-6). In 2003, Kamisawa et al (3) proposed the new clinicopathological entity of "IgG4-related autoimmune disease" based on common pathological features of many IgG 4-positive plasma cell infiltrates with fibrosis and increased serum IgG4 levels, which are representative findings of autoimmune pancreatitis. Since then, many case reports or case series have accumulated, and IgG4-related disease has been accepted as a new clinical entity. IgG4-related chronic sclerosing dacryoadenitis and sialoadenitis are major components of this disease.

However, only a few reports have focused on the skin lesions associated with autoimmune pancreatitis, chronic sclerosing dacryoadenitis and sialoadenitis or systemic IgG4related lymphoadenopathy $(7,8)$. Here, we describe a case of IgG4-related chronic sclerosing dacryoadenitis and sialoadenitis with nodular skin lesions with marked IgG4positive plasma cell infiltration and scattered eosinophil infiltration, which appeared in parallel with exacerbation of

\footnotetext{
${ }^{1}$ Division of Rheumatology, Department of Internal Medicine, Kanazawa University Graduate School of Medicine, Japan, ${ }^{2}$ Division of Cardiology, Department of Internal Medicine, Kanazawa University Graduate School of Medicine, Japan, ${ }^{3}$ Research Center for Medical Education, Kanazawa University Graduate School of Medicine, Japan, ${ }^{4}$ Hematology and Immunology, Kanazawa Medical University, Japan, ${ }^{5}$ Institute of Liver Studies, King's College Hospital, UK and ${ }^{6}$ Department of Dermatology, Kanazawa University Graduate School of Medical Science, Japan Received for publication January 31, 2011; Accepted for publication March 23, 2011 Correspondence to Dr. Mitsuhiro Kawano, sk33166@gmail.com
} 

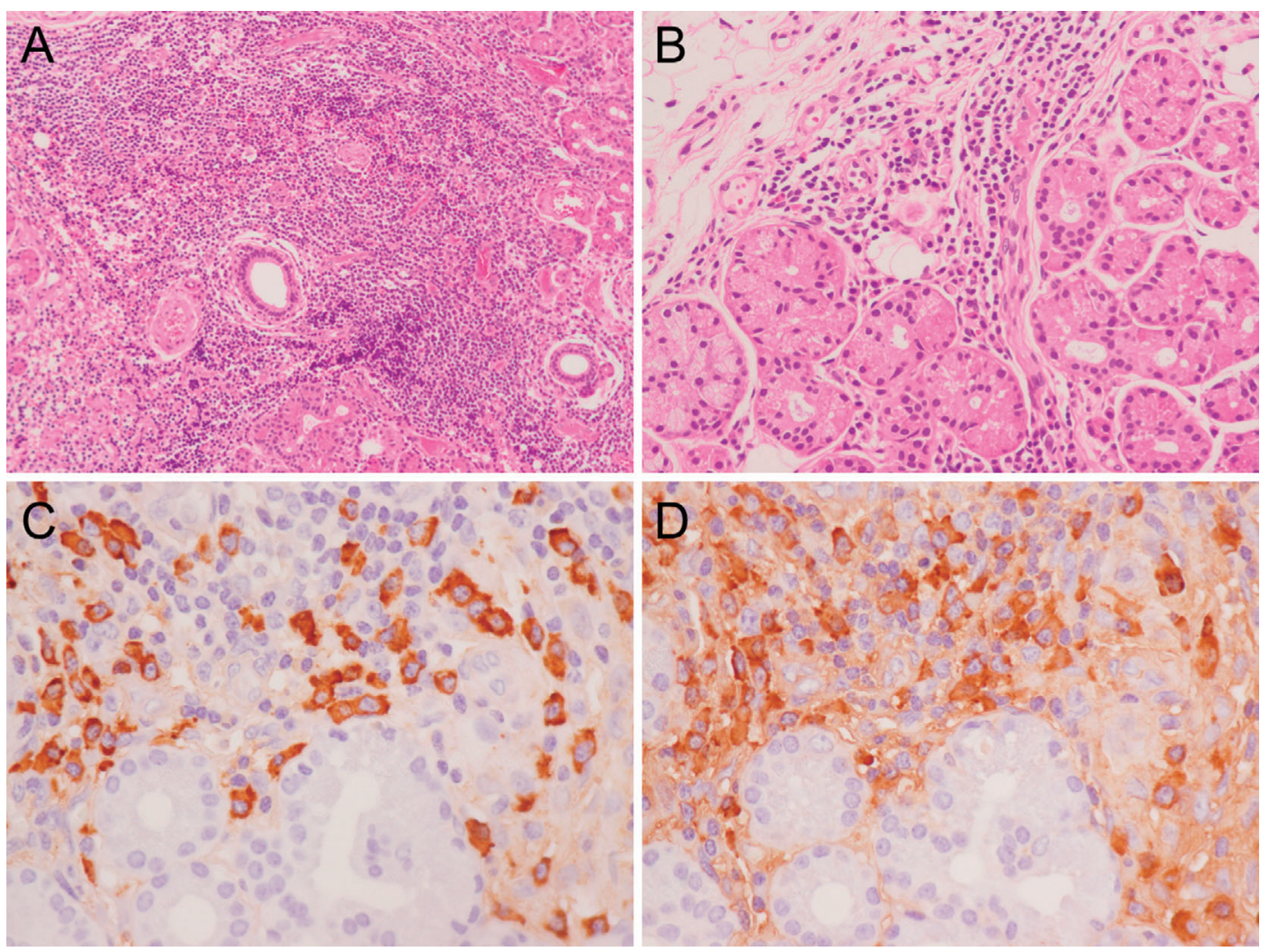

Figure 1. Lacrimal gland biopsy shows marked infiltration of lymphocytes and plasma cells (A) with mild fibrosis (B). Many infiltrating plasma cells are IgG4 positive (C), with an IgG4/IgG ratio of $84.8 \%$ (C and D). [(A) lacrimal gland, Hematoxylin and Eosin (HE) staining, $\times 100$, (B) HE staining, $\times 200$, (C) IgG4, ×400, (D) IgG, ×400]

the dacryoadenitis and improved after the corticosteroid dosage was increased.

\section{Case Report}

A 60-year-old man was admitted to our hospital for close examination of impaired glucose tolerance and systemic evaluation of IgG4-related disease. One year before entry, a high fasting plasma glucose level had been pointed out for the first time on an annual health checkup and he began treatment for diabetes mellitus. Six months before admission, he noticed protrusion of his left eye, and two months later itchy nodules on his scalp and neck. Magnetic resonance imaging revealed left external eye muscle hypertrophy and multiple mass lesions in the left orbital cavity. As malignant lymphoma was strongly suspected, a left lacrimal gland biopsy was performed. The biopsy specimen was composed of inflammatory tissue with marked infiltrates of IgG4-positive plasma cells and scattered eosinophilic infiltrates with fibrosis suggesting IgG4-related disease (Fig. 1A, 1B). The average ratio of $\mathrm{IgG} 4 / \mathrm{IgG}$ positive plasma cells in five different high power fields (hpf) with intense infiltration was $84.8 \%$ (Fig. 1C, 1D). On admission to our hospital, physical examination revealed left eye extrusion with obvious lacrimal gland swelling (Fig. 2A). Small itchy nodules were found on the parietal scalp, and 7 little finger tip-sized itchy subcutaneous erythematous nodules on the neck without any palpable lymph nodes (Fig. 2B). The bilateral parotid glands were swollen, while the submandibular glands were of normal size. He had no history of allergies. Blood eosinophil count was $993 / \mathrm{mL}$ accounting for $12.9 \%$ of the total white blood cell count. Fasting plasma glucose was $100 \mathrm{mg} / \mathrm{dL}$, and $\mathrm{HbAlc}$ 6.4\%. Liver function tests, electrolytes, and renal function tests were all within the respective normal ranges. Serum $\operatorname{IgG}$ level was 1,570 $\mathrm{mg} / \mathrm{dL}$, IgG4 $463 \mathrm{mg} / \mathrm{dL}(29.5 \%$ ), and $\mathrm{IgE} 4,554 \mathrm{IU} / \mathrm{mL}$, rheumatoid factor $12 \mathrm{IU} / \mathrm{mL}$, and soluble interleukin 2 receptor $692 \mathrm{U} / \mathrm{mL}$ (normal 220-530 U/mL). Antinuclear antibodies were negative. Computed tomography (CT) scans revealed bilateral lacrimal gland and parotid gland swelling without lymphadenopathy. Abdominal CT showed a normalsized pancreas without pancreatic duct abnormalities or mass formation. A skin biopsy of a cutaneous nodule was performed. On light microscopy, there was moderate lymphocyte and plasma cell infiltration around arterioles and adnexal structures in the dermis (Fig. 3A). In particular, severe lymphocytic infiltration with plasma cells and eosinophils around arterioles or venules was evident in the deep dermis (Fig. 3B, 3C) and subcutaneous fat tissue, and the majority of infiltrating plasma cells were IgG4 positive (average IgG4 positive cell count in five different hpf with intense infiltration was $47 / \mathrm{hpf}$ ) (Fig. 3D). A diagnosis of IgG 4-related systemic disease was made because of an elevated serum level of $\operatorname{IgG} 4$, marked infiltration of $\operatorname{IgG} 4$-positive 

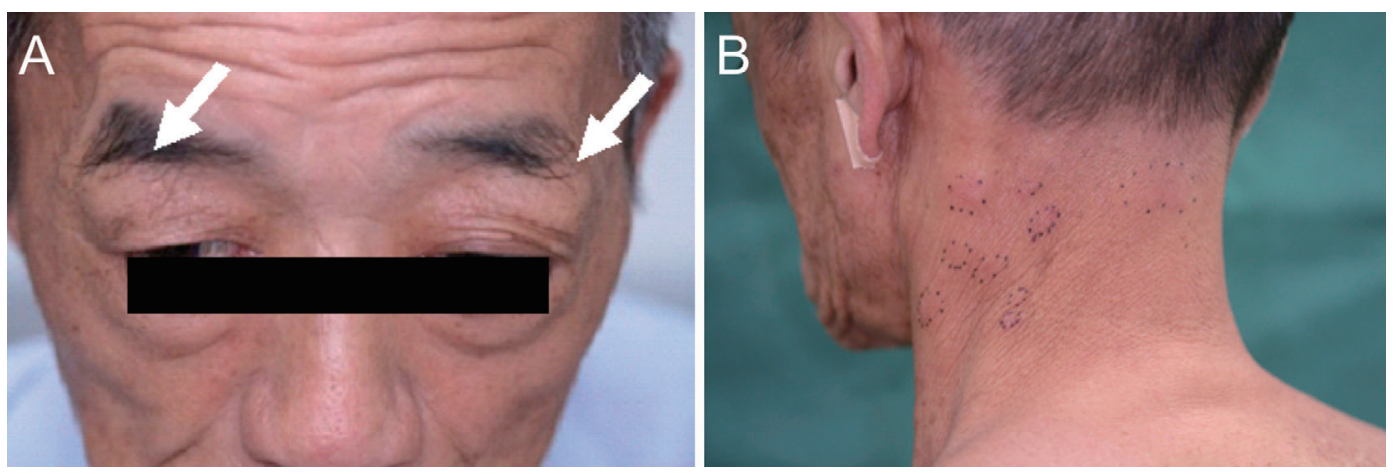

Figure 2. Bilateral swelling of the lacrimal glands is noted (A, arrows). Little finger tip-sized subcutaneous erythematous nodules are present on the neck $(B)$.

plasma cells in the lacrimal glands, and typical features of Mikulicz's disease with symmetrical lacrimal and parotid gland swelling. After the administration of $20 \mathrm{mg}$ of prednisolone, a rapid response was obtained and the multiple nodules in the scalp and neck disappeared. The bilateral parotid swelling was also improved. The prednisolone dose was reduced at the rate of $5 \mathrm{mg}$ every two weeks to $10 \mathrm{mg}$, which was adopted as the maintenance dose. Six months thereafter, the left eye protrusion, bilateral parotid swelling, and multiple subcutaneous nodules recurred, and the dose of prednisolone was increased to $20 \mathrm{mg}$ after a second skin biopsy. The histopathological findings were similar to those of the previous biopsy with marked IgG4-positive plasma cell infiltration with scattered eosinophils, supporting the recurrence of IgG4-related disease. Twenty days after readministration of $20 \mathrm{mg}$ of prednisolone, ${ }^{18} \mathrm{~F}$-fluorodeoxyglucose positron emission tomography (FDG-PET) was performed. However, no FDG-PET positive lesion was detected, this being consistent with the rapidly improved clinical findings. After that, prednisolone was carefully decreased without recurrence of the eye protrusion, parotid swelling, or appearance of new skin lesions.

\section{Discussion}

We report a patient with clinical and histological features of chronic sclerosing dacryoadenitis and sialoadenitis and multiple nodular itchy skin lesions on the scalp and neck. The histological findings of the skin lesions were very similar to those previously reported in IgG4-related disease $(3-5,8)$, suggesting that the skin lesions of this case should be included as one of the extra-pancreatic manifestations of autoimmune pancreatitis and other IgG4-related disease.

To identify new organ involvement of IgG4-related disease, two approaches to identification exist. One is to find marked IgG4-positive plasma cell infiltration in a suspicious lesion, and to confirm an elevated serum IgG4 level. The other is to find an associated lesion in patients with typical IgG4-related disease, such as autoimmune pancreatitis or IgG4-related chronic sclerosing dacryoadenitis and sialoadenitis, and to prove similar IgG4-positive plasma cell infiltra- tion in the newly recognized lesion. However, the former approach has not yet been fully accepted because some patients with well established diseases such as Churg-Strauss syndrome (9) and Castleman's disease $(6,10,11)$ also have similar IgG4-positive plasma cell infiltration with high serum IgG4 levels.

Kuo et al (12) contended that cutaneous Rosai-Dorfman (RD) disease is an IgG4-related sclerosing disease according to identification by the former approach. They analyzed the skin lesions of 12 patients with RD disease, and noted that all but one of them had more than $30 \mathrm{IgG} 4$ positive cells/ hpf. They also found an elevated serum IgG4 level in one patient. Shrestha et al (13) analyzed lung lesions of 8 patients with nodal and extranodal RD disease, and found that 6 of 8 RD cases showed an increased number of IgG4positive plasma cells in the lung. Although these findings suggest that some relationship may exist between RD disease and IgG4-related disease, the finding of S-100-proteinpositive large histiocytes, a histopathological feature of RD disease, is very unusual in IgG4-related disease, making it difficult to regard cutaneous RD disease as a cutaneous manifestation of IgG4-related disease. However, Shrestha et al (13) showed that 2 of 6 patients with lung lesions associated with IgG4-related autoimmune pancreatitis had prominent lymphatic dilatation with emperipolesis and S-100 protein-positive histiocytes in the lung. Therefore, further studies are needed to classify RD disease as an IgG4-related disease.

Miyagawa-Hayashino et al (14) claimed that cutaneous plasmacytosis is a cutaneous manifestation of IgG4-related disease as identified by the former approach. Although hypergammaglobulinemia is common to both cutaneous plasmacytosis and IgG4-related disease, an elevated serum interleukin 6 (IL-6) level, which is a common feature of cutaneous plasmacytosis (15), is very uncommon in IgG4-related disease (7). In addition, an association of pancreatic, lacrimal or salivary gland lesions with cutaneous plasmacytosis has not been reported previously. Therefore, careful judgment is needed to classify cutaneous plasmacytosis as an IgG4-related disease.

In contrast, the present case showed that skin might also be involved in IgG4-related disease as identified by the lat- 

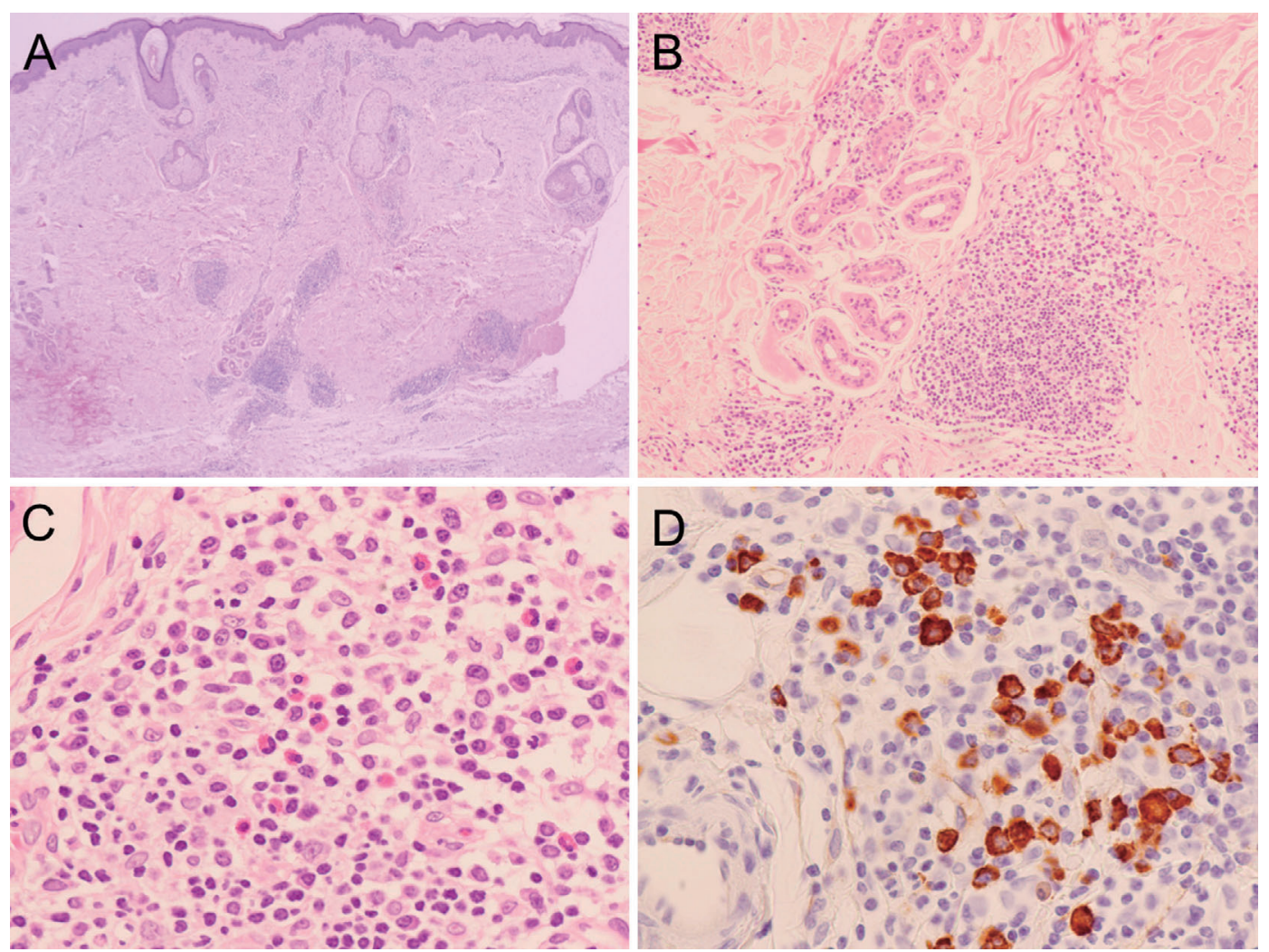

Figure 3. Moderate infiltration of lymphocytes and plasma cells is noted around arterioles or venules in the deep dermis and subcutaneous fat tissue (A). Marked lymphocyte and plasma cell infiltration is noted in the deep dermis without evident fibrosis (B). There are many infiltrating eosinophils (C). Many IgG4-positive plasma cell infiltrates in the skin lesion are seen [average IgG4 positive cell count in five different high power fields (hpf) with intense infiltration: 47/hpf] (D). [(A) skin, Hematoxylin and Eosin (HE) staining, $\times 40$, (B) HE staining, $\times 100$, (C) HE staining, $\times 400,(D)$ IgG4, ×400]

ter approach. In our case, the histological findings of the skin lesions with eosinophil infiltration were very similar to those of AIP or IgG4-related chronic sclerosing dacryoadenitis or sialoadenitis $(3,5,6)$. Moreover, the skin, lacrimal, and parotid lesions deteriorated simultaneously during steroid tapering and improved after increasing the dosage of corticosteroid, suggesting a similar pathophysiological involvement in these organs.

Only two papers referring to the skin lesions of IgG4related disease identified by the latter approach are available, but the clinical features of the skin lesions were not fully described and their response to corticosteroid therapy was not mentioned in detail. Sato et al (7) showed that 3 of 9 patients with systemic IgG4-related lymphoadenopathy had skin lesions, and demonstrated that one of them had cutaneous pathological findings typical of IgG4-related disease. However, macroscopic findings and the distribution of the skin lesions were not shown in their paper, making it difficult to compare their lesions with those of the present case. Cheuk et al (8) proposed that cutaneous pseudolymphoma might be a skin manifestation of IgG4-related sclerosing disease. Their two cases had lacrimal or salivary gland lesions with markedly elevated serum $\operatorname{IgG} 4$ levels. The cutaneous lesions of the present case were itchy and erythematous, which were consistent with those of their case. The distribution of the skin lesions in the scalp, face and neck was also very similar in their cases and ours. Although the histological findings had many similarities, our patient did not have evident fibrosis with Azan stain (data not shown) and showed less marked lymphocyte and plasma cell infiltration than their cases with pseudolymphoma formation. Therefore, we speculate that the lesion in our case was of an earlier stage than that described by Cheuk et al (8), and that our case if left untreated might also develop a similar pseudolymphoma in the future.

Further study of the skin manifestations of IgG4-related disease is needed so as to enhance our understanding of the clinical spectrum, pathophysiology and response to therapy of this disorder.

\section{The authors state that they have no Conflict of Interest (COI).}

\section{Acknowledgement}

We would like to thank John Gelblum for his critical reading of the manuscript. This work was supported in part by grants from the Ministry of Health, Labor, and Welfare of Japan (HU and MK). 


\section{References}

1. Finkelberg DL, Sahani D, Deshpande V, Brugge WR. Autoimmune pancreatitis. N Engl J Med 355: 2670-2676, 2006.

2. Okazaki K, Kawa S, Kamisawa T, et al. Clinical diagnostic criteria of autoimmune pancreatitis: revised proposal. J Gastroenterol 41: 626-631, 2006.

3. Kamisawa T, Funata N, Hayashi Y, et al. A new clinicopathological entity of IgG4-related autoimmune disease. J Gastroenterol 38: 982-984, 2003.

4. Kamisawa T, Okamoto A. IgG4-related sclerosing disease. World J Gastroenterol 14: 3948-3955, 2008.

5. Masaki Y, Dong L, Kurose N, et al. Proposal for a new clinical entity, IgG4-positive multiorgan lymphoproliferative syndrome: analysis of 64 cases of IgG4-related disorders. Ann Rheum Dis 68: 1310-1315, 2009.

6. Zen Y, Nakanuma Y. IgG4-related disease: a cross-sectional study of 114 cases. Am J Surg Pathol 34: 1812-1819, 2010.

7. Sato Y, Kojima M, Takata K, et al. Systemic IgG4-related lymphadenopathy: A clinical and pathologic comparison to multicentric Castleman's disease. Mod Pathol 22: 589-599, 2009.

8. Cheuk W, Lee KC, Chong LY, Yuen ST, Chan JKC. IgG4-related sclerosing disease: A potential new etiology of cutaneous pseudolymphoma. Am J Surg Pathol 33: 1713-1719, 2009.
9. Yamamoto M, Takahashi H, Suzuki C, et al. Analysis of serum IgG subclasses in Churg-strauss syndrome-the meaning of elevated serum levels of IgG4. Intern Med 49: 1365-1370, 2010.

10. Miwa I, Maruyama Y, Kageoka M, et al. Retroperitoneal fibrosis and Castleman disease in two patients with high IgG4 levels. Nippon Shokakibyo Gakkai Zasshi 105: 1087-1092, 2008 (in Japanese).

11. Sato Y, Kojima M, Takata K, et al. Multicentric Castleman's disease with abundant IgG4-positive cells: a clinical and pathological analysis of six cases. J Clin Pathol 63: 1084-1089, 2010.

12. Kuo TT, Chen TC, Lee LY, Lu PH. IgG4-positive plasma cells in cutaneous Rosai-Dorfman disease: an additional immunohistochemical feature and possible relationship to IgG4-related sclerosing disease. J Cutan Pathol 36: 1069-1073, 2009.

13. Shrestha B, Sekiguchi H, Colby TV, et al. Distinctive pulmonary histopathology with increased IgG4-positive plasma cells in patients with autoimmune pancreatitis: report of 6 and 12 cases with similar histopathology. Am J Surg Pathol 33: 1450-1462, 2009.

14. Miyagawa-Hayashino A, Matsumura Y, Kawakami F, et al. High ratio of $\mathrm{IgG} 4$-positive plasma cell infiltration in cutaneous plasmacytosis--is this a cutaneous manifestation of IgG4-related disease? Hum Pathol 40: 1269-1277, 2009.

15. Yamamoto T, Katayama I, Nishioka K. Increased plasma interleukin-6 in cutaneous plasmacytoma: the effect of intralesional steroid therapy. Br J Dermatol 137: 631-636, 1997.

(C) 2011 The Japanese Society of Internal Medicine

http://www.naika.or.jp/imindex.html 